\title{
EPMA, EPR, electronic and vibrational spectral studies on natural aurichalcite
}

\author{
S VEDANAND, B MADHU SUDHANA, B J REDDY* and \\ P SAMBASIVA RAO ${ }^{+}$ \\ Department of Physics, S V University. Tirupati 517 502, India \\ 'Department of Chemistry, Pondicherry University, Pondicherry 605014, India
}

MS received 20 September 1994; revised 20 October 1995

\begin{abstract}
The electron probe micro analysis (EPMA), electron paramagnetic resonance (EPR), electronic and vibrational spectral studies on a natural mineral, aurichalcite were studied at room temperature. The EPMA analysis revealed the concentration of copper in the mineral to be $2.6 \mathrm{wt} \%$ and zinc as $24.5 \mathrm{wt} \%$. The optical spectra revealed the presence of copper in $D_{4 \mathrm{~h}}$ symmetry with crystal field $(D q)$ and tetragonal field (Ds and $D t$ ) parameters as 1250,1600 and $520 \mathrm{~cm}^{-1}$ respectively. This further confirmed the presence of $\mathrm{Cu}(\mathrm{II})$ ion in an elongated tetragonal site. The EPR studies indicated the presence of $\mathrm{Cu}(\mathrm{II})$ ion, but hyperfine lines could not be resolved due to the high concentration of the paramagnetic impurity in the mineral. The IR spectrum confirmed the presence of water and carbonate ion.
\end{abstract}

Keywords. Aurichalcite; optical absorption spectra; EPR; $\mathrm{Cu}(\mathrm{II})$ ion.

\section{Introduction}

The $\mathrm{Cu}(\mathrm{II})$ is a transition metal ion that readily forms stable complexes. It has a very simple electronic configuration, namely one electron is missing from the complete $\mathrm{d}$ shell. The system is equivalent to a single positive charge in the $3 \mathrm{~d}$-shell. Hence, it is a very convenient system for checking some of the basic ideas of the crystal field theory in naturally occurring minerals. In this connection, a few copper bearing minerals have been investigated to explain the nature of the metal ion and its site symmetry by spectroscopic measurements (Reddy and Sarma 1981; Sarma et al 1982; Reddy et al 1987).

The chemical composition of copper bearing aurichalcite mineral is $\mathrm{ZnCu}\left(\mathrm{CO}_{3}\right)(\mathrm{OH})_{2}$. In this mineral, copper and zinc are mutually replaceable and are surrounded by two $\mathrm{O}$ and four $\mathrm{OH}$ molecules in a less distorted octahedron. Crystal structure and chemical analysis of aurichalcite from different origins have been reported (Jambor and Pouliot 1965; Zirkl 1970; Leicht 1971; Palache et al 1976).

The present investigation is on the greenish blue mineral aurichalcite, collected from Banner district, dripping spring mountain, Gila Country, Arizona, USA supplied by WARDS Natural Science Estd., Inc., New York. Since, copper(II), being a $\mathrm{d}^{\mathbf{9}}$ system, is prone to Jahn-Teller distortion, an EPR study of this ion will give information about the type of distortion. Hence, IR and EPR techniques have been used in the present study to confirm the symmetry around the paramagnetic ion in the mineral at room temperature.

\footnotetext{
*Author for correspondence
} 


\section{Experimental}

Microanalysis was carried out on thin polished section of aurichalcite using Camebax Micro EPMA at an operating voltage of $20 \mathrm{kV}$ and a sample current of $2 \mathrm{n}$.amp. in a beam regulated mode. The measured data was corrected by ZAF correction on PDP $11 / 03$ computer. Optical absorption spectrum of the sample in the form of powder was recorded on Cary 2390 spectrometer in the region 300 to $1200 \mathrm{~nm}$. EPR spectrum of the sample in polycrystalline form was recorded using Varian E-112 EPR spectrometer operating at $X$-band frequencies $(v=9.5 \mathrm{GHz})$ having a $100 \mathrm{kHz}$ field modulation and a phase sensitive detector to obtain a first derivative signal. DPPH with a $g$ value of 2.0036 was used as the internal field marker. The infrared spectrum of the sample in $\mathrm{KBr}$ pellet form was recorded on Perkin-Elmer infrared spectrophotometer in the region 600 to $4000 \mathrm{~cm}^{-1}$.

\section{Results and discussion}

\subsection{Chemical composition}

Elemental concentration of aurichalcite from EPMA is presented in table 1. Ideally pure aurichalcite has the structural formula $\mathrm{ZnCu}\left(\mathrm{CO}_{3}\right)(\mathrm{OH})_{2}$. However, the analysis indicates the presence of Si varying from 13 to $18 \mathrm{wt} \%$. This might be due to silica in the associated silicate of the rock detected by the probe. Table 1 shows that this mineral contains $\mathrm{Zn}$ to the extent of 24 to $28 \mathrm{wt} \%$ while the concentration of $\mathrm{Cu}$ varies from $2 \cdot 4$ to $2.8 \mathrm{wt} \%$. X-ray scanning picture is taken for $\mathrm{MnK}_{\alpha}$ line on the sample which revealed the presence of $\mathrm{Mn}$ in very low concentration and this could not be estimated.

\subsection{Optical absorption spectrum}

The optical absorption spectrum of the sample is shown in figure 1. Since the spectrum consists of three bands, the site symmetry of $\mathrm{Cu}(\mathrm{II})$ is presumed to be $D_{4 \mathrm{~h}}$. Accordingly, the three bands observed at 9010,12500 and $14700 \mathrm{~cm}^{-1}$ are attributed to the transitions ${ }^{2} B_{1 \mathrm{~g}} \rightarrow{ }^{2} A_{1 \mathrm{~g}},{ }^{2} B_{1 \mathrm{~g}} \rightarrow{ }^{2} B_{2 \mathrm{~g}}$ and ${ }^{2} B_{1 \mathrm{~g}} \rightarrow{ }^{2} E_{\mathrm{g}}$ respectively. Therefore the energy expressions can be written as

$$
\begin{array}{r}
4 D s+5 D t=9010 \mathrm{~cm}^{-1} \\
10 D q=12500 \mathrm{~cm}^{-1} \\
10 D q+3 D s-5 D t=14700 \mathrm{~cm}^{-1}
\end{array}
$$

Table 1. EPMA data of aurichalcite.

\begin{tabular}{lrrrrrr}
\hline & \multicolumn{5}{c}{ Concentration in wt\% (sets) } \\
Element & \multicolumn{1}{c}{ I } & \multicolumn{1}{c}{ II } & III & IV & V & VI \\
\hline Cu & 2.41 & 2.76 & 2.83 & 2.60 & 2.46 & 2.53 \\
$\mathrm{Zn}$ & 23.74 & 25.41 & 28.19 & 24.86 & 26.86 & 24.51 \\
$\mathrm{Si}$ & 18.39 & 17.79 & 17.71 & 18.19 & 12.87 & 16.84 \\
\hline
\end{tabular}




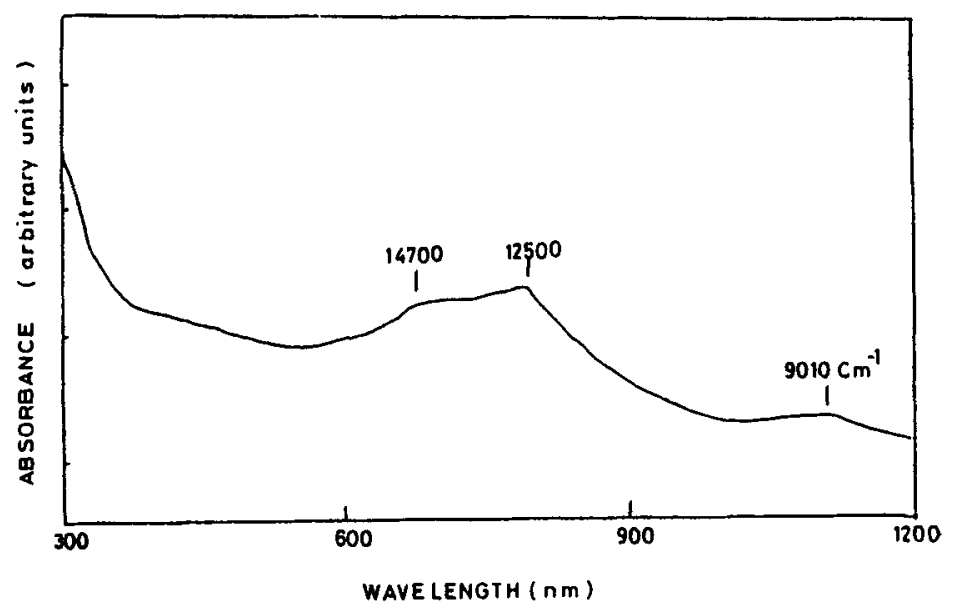

Figure 1. Room temperature optical absorption spectrum of aurichalcite in powder form.

Table 2. Comparison of crystal field parameters of six-coordinated compounds.

\begin{tabular}{lcccc}
\hline Compound & $D q$ & $D s$ & $D t$ & Ref. \\
\hline $\mathrm{Cu}\left(\mathrm{HCO}_{2}\right)_{2} \cdot 4 \mathrm{H}_{2} \mathrm{O}$ & 1120 & 1600 & 560 & {$[\mathrm{a}]$} \\
$\mathrm{Cu}^{2+}: \mathrm{CaC}_{4} \mathrm{H}_{4} \mathrm{O}_{6} \cdot 4 \mathrm{H}_{2} \mathrm{O}$ & 1000 & 1540 & 470 & {$[\mathrm{~b}]$} \\
$\mathrm{Cu}_{8}\left(\mathrm{Si}_{4} \mathrm{O}_{11}\right) \mathrm{OH}_{4}$ & 1070 & 1674 & 327 & {$[\mathrm{c}]$} \\
$\mathrm{Cu}_{5}\left(\mathrm{SiO}_{3}\right)_{4} \cdot \mathrm{OH}_{2}$ & 1333 & 1565 & 380 & {$[\mathrm{c}]$} \\
$\mathrm{Cu}_{19}\left(\mathrm{SO}_{4}\right) \mathrm{Cl}_{4}(\mathrm{OH})_{32} \cdot 3 \mathrm{H}_{2} \mathrm{O}$ & 1205 & 1525 & 450 & {$[\mathrm{~d}]$} \\
$\mathrm{ZnCu}\left(\mathrm{CO}_{3}\right)(\mathrm{OH})_{2}$ & 1250 & 1600 & 520 & Present case \\
\hline
\end{tabular}

[a] Billing and Hathway (1968); [b] Swamy et al (1980); [c] Sarma et al (1982); [d] Sreeramulu et al (1990).

Using the formulae of the transitions, the crystal field $(D q)$ and tetragonal field $(D s$ and $D t$ ) parameters are calculated as $D q=1250, D s=1600$ and $D t=520 \mathrm{~cm}^{-1}$. In tetragonal field the values of $D t$ and $D q$ will have the same sign if there is an axial elongation and will have opposite sign if they have axial compression (Ferguson et al 1975). For the present case the values of $D t$ and $D q$ are found to have the same sign. This confirms the lowering of octahedral site to an elongated tetragonal site of $\mathrm{Cu}$ (II) ion in the crystal. A comparison of the parameters $D q, D s$ and $D t$ of certain copper samples in tetragonal site (symmetries) is made (table 2). The present one agrees with those reported earlier.

\subsection{EPR spectrum}

The mineral was taken in a mortar and with a pestle was made into a fine powder. The powdered mineral was then transferred into an EPR quartz tube and the EPR spectrum was recorded at room temperature. The spectrum thus obtained is shown in 


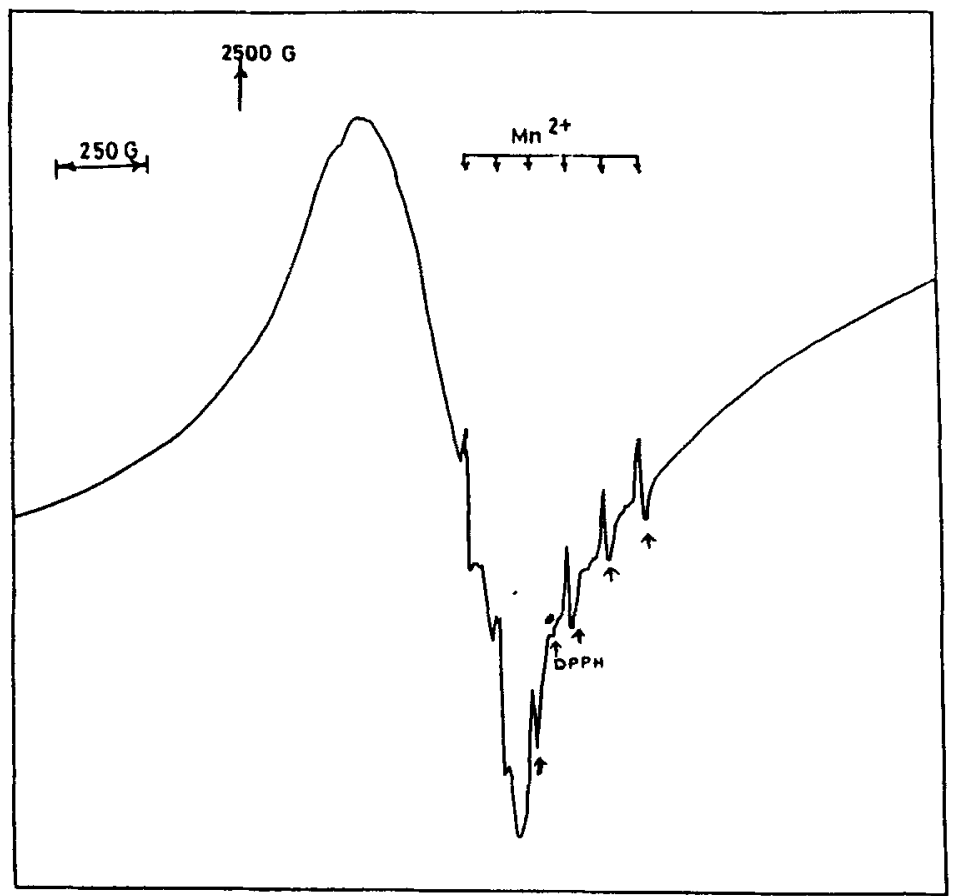

Figure 2. EPR spectrum of polycrystalline sample of aurichalcite at room temperature. Frequency $=9.501 \mathrm{GHz}$.

figure 2. It is clear from figure 2 that it contains a broad peak and a sextet pattern of sharp lines marked by arrows from the top. The line marked with * is due to the internal standard DPPH. The broad line has a $\mathrm{g}$ value of $2 \cdot 28$, which can be assigned to a $\mathrm{Cu}$ (II) ion in the mineral. Copper nucleus has two isotopes with mass numbers 63 and 65 . Both these isotopes have nuclear spin of $3 / 2$ and also have almost identical magnetic moments (Weil et al 1994). As was mentioned in optical absorption spectrum analysis, copper ion undergoes elongated tetragonal distortion. Hence, one should expect more than one $g$ value in the EPR spectrum. In the present case, one $g$ value is 2.28 and the other $\mathrm{g}$ value, which will be around $2 \cdot 06$, was hidden under the broad line. In addition to this, since the concentration of copper is high (around $2.6 \mathrm{wt} \%$ ), the hyperfine lines from the nuclear spin of copper nucleus could not be resolved due to dipolar-dipolar broadening.

The sharp six lines marked by arrows from the top, have a $\mathrm{g}^{-}$value of 2.002 and a hyperfine coupling constant of $95(5) \mathrm{G}$. These values are typical for a $\mathrm{Mn}(\mathrm{II})$ ion impurity in the lattice (Rao 1993). Generally one would expect a sextet for $\mathrm{Mn}$ (II) in polycrystalline form, because the other four electron spin transitions (for a divalent manganese ion, total spin $S=5 / 2$ ), that are $| \pm 5 / 2\rangle \leftrightarrow| \pm 3 / 2\rangle$ and $| \pm 3 / 2\rangle \leftrightarrow| \pm 1 / 2\rangle$ have very large anisotropy and hence often difficult to observe (Blanchard and Chasteen 1976). Also, the value of the zero-field splitting parameter indicates the number of lines (Sambasiva Rao 1993). The low intensity of these six lines indicate the low percentage of manganese in the mineral sample. The broad line under the sextet may be due to copper perpendicular $g$ value, as mentioned above. Cooling the sample to $77 \mathrm{~K}$ does not change the general nature of the spectrum. 


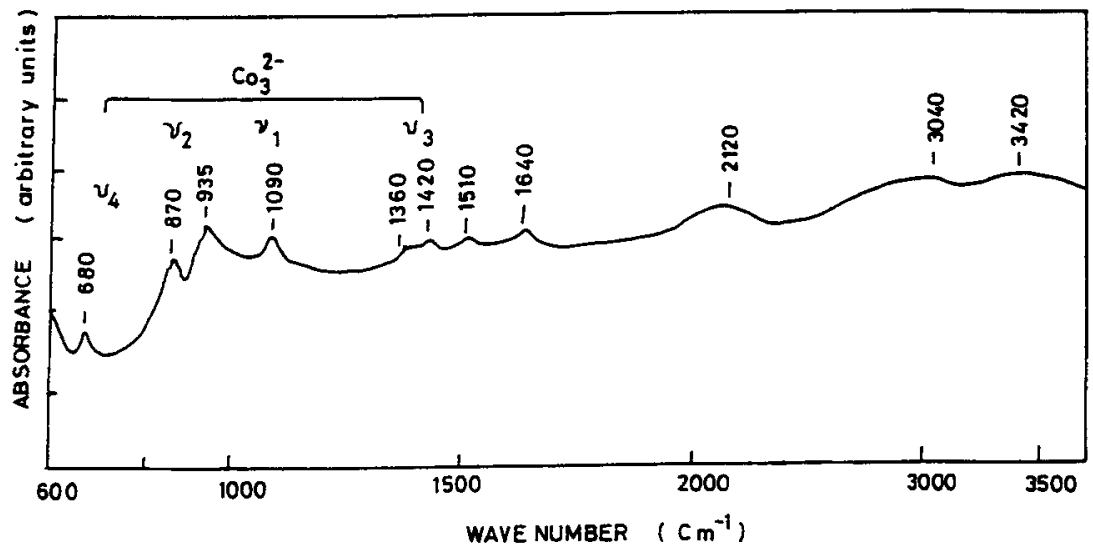

Figure 3. Infrared spectrum of aurichalcite in $\mathrm{KBr}$ pellet at room temperature.

\subsection{Vibrational spectrum}

Infrared spectrum of the sample recorded in the region 600 to $4000 \mathrm{~cm}^{-1}$ is shown in figure 3. The four groups of bands observed around $680,900,1090$ and $1400 \mathrm{~cm}^{-1}$ are identified as the fundamental frequencies of $\mathrm{CO}_{3}^{2-}$ namely $v_{4}, v_{2}, v_{1}$ and $v_{3}$. Among these, symmetry stretching mode $v_{1}$ alone is infrared inactive. However, in the case of carbonate compounds, $v_{1}$ can also be observed and hence the $1090 \mathrm{~cm}^{-1}$ band is identified as $v_{1}$. The bands at 870 and $935 \mathrm{~cm}^{-1}$ are due to out of plane bending mode $v_{2}$, whereas the bands at 1360,1420 and $1510 \mathrm{~cm}^{-1}$ are due to asymmetric stretching mode $v_{3}$. The in-plane bending mode $v_{4}$ is assigned to the band at $680 \mathrm{~cm}^{-1}$. The bands at $1640,3040 \mathrm{~cm}^{-1}$ are identified as due to water, the $\mathrm{H}-\mathrm{O}-\mathrm{H}$ bending $v_{2}$ and $\mathrm{OH}$ symmetric stretching $v_{1}$ respectively, whereas the other band at $3420 \mathrm{~cm}^{-1}$ is due to asymmetric OH stretching (Reddy et al 1986). The band observed at $2120 \mathrm{~cm}^{-1}$ might be due to low energy $\mathrm{OH}$ valence vibrations (Langer and Raith 1974).

\section{Conclusion}

Optical absorption spectrum of aurichalcite indicates that the replacement of $\mathrm{Zn}$ by $\mathrm{Cu}$ is in accordance with the crystal structure reported. The crystal field and tetragonal field parameters confirm further the lowering of octahedral site to an elongated tetragonal $\left(D_{4 \mathrm{~h}}\right)$ site of $\mathrm{Cu}(\mathrm{II})$ ion in the crystal.

The EPR spectrum indicates the presence of copper (II) in a lower symmetry. The dipolar-dipolar broadening made the hyperfine lines unresolved and the second $\mathrm{g}$ value for $\mathrm{Cu}(\mathrm{II})$ is hidden under the $\mathrm{Mn}$ (II) sextet (lower field side). The EPR analysis also indicates the presence of $\mathrm{Mn}$ (II) in traces, which was further confirmed by X-ray scanning picture for $\mathrm{MnK}_{\alpha}$ line on the sample. This study indicates the significance of EPR technique to measure paramagnetic impurities present in traces in natural minerals, simultaneously with another paramagnetic impurity present in higher levels. The vibrational spectrum of the minerals exhibits the fundamental frequencies of water and carbonate ion. 


\section{Acknowledgements}

The authors thank UGC, New Delhi for sponsoring the project. One of the authors (BMS) thanks CSIR, New Delhi for awarding SRF.

\section{References}

Blanchard S C and Chasteen N D 1976 J. Phys. Chem. 801362

Billing D E and Hathway B J $1968 \mathrm{~J}$. Chem.-Soc. (A) 1516

Ferguson J, Wood T E and Guggenheim H J 1975 Inorg. Chem. 14177

Jambor J L and Pouliot G 1965 Can. Min. 8385

Langer K and Raith M 1974 Am. Mineral. 591249

Leicht W C 1971 Mineral. Rec. 2214

Palache C, Berman H and Frondel C 1976 in The system of mineralogy (New York: John Wiley \& Sons) 2249

Reddy B J and Sarma K B N 1981 Solid State Commun. 38547

Reddy K M, Jacob A S and Reddy B J 1986 Phys. Lett. A115 182

Reddy K M, Jacob A S, Reddy B J and Reddy Y P 1987 Phys. Status Solidi 139 K 145

Sambasiva Rao P 1993 Spectrochimica Acta A49 897

Sarma K B N, Reddy B J and Lakshman S V J 1982 Phys. Lett. A92 305

Sreeramulu P, Reddy K M, Jacob A S, Reddy B J and Reddy Y P 1990 J. Cryst. Spectroscopic Res. 2093

Swamy Y K R, Reddy P P and Reddy Y P 1980 Physica $\mathbf{B 8 9} 202$

Weil J A, Bolton J R and Wertz J E 1994 Electron paramagnetic resonance; Elementary theory and practical applications (New York: Academic Press) p. 27

Zirkl E J 1970 in Mitteilungsbl. Mus. Burgbau. Geol. Peleontol. Landesmus Joameum Abt. Mineral. 139 